\title{
Endogenous Leu332GIn mutation in p53 disrupts the tetramerization ability in a canine mammary gland tumor cell line
}

\author{
KAZUHIKO OCHIAI $^{*}$, DAIGO AZAKAMI ${ }^{1 *}$, MASAMI MORIMATSU ${ }^{2}$, HINAKO HIRAMA $^{1}$, \\ SHOTA KAWAKAMI ${ }^{1}$, TAKAYUKI NAKAGAWA ${ }^{3}$, MASAKI MICHISHITA ${ }^{4}$, AI S. EGUSA ${ }^{5}$, \\ TAKANORI SASAKI $^{6}$, MASAMI WATANABE ${ }^{6}$ and TOSHINORI OMI ${ }^{1}$
}

\begin{abstract}
${ }^{1}$ School of Veterinary Nursing and Technology, Faculty of Veterinary Science, Nippon Veterinary and Life Science University,
Tokyo 180-8602; ${ }^{2}$ Laboratory of Laboratory Animal Science and Medicine, Department of Disease Control, Graduate School of Veterinary Medicine, Hokkaido University, Sapporo 060-0818; ${ }^{3}$ Department of Veterinary Surgery, The University of Tokyo, Bunkyo, Tokyo 113-8657; ${ }^{4}$ Department of Veterinary Pathology, Faculty of Veterinary Science,

Nippon Veterinary and Life Science University; ${ }^{5}$ Department of Applied Life Science,

Faculty of Food Science, Nippon Veterinary and Life Science University, Tokyo 180-8602;

${ }^{6}$ Department of Urology, Graduate School of Medicine, Dentistry and Pharmaceutical Sciences, Okayama University, Okayama 700-8558, Japan
\end{abstract}

Received December 22, 2017; Accepted April 27, 2018

DOI: 10.3892/or.2018.6409

\begin{abstract}
Mutations in the $p 53$ gene are associated with more than half of all human cancers. These mutations often cause a disruption of the tumor-suppressor function of $\mathrm{p} 53$ and induce genomic instabilities. Wild-type p53 requires tetramerization to function as an initiator of cell cycle arrest and apoptosis. Although alterations in p53 tetramerization caused by mutation have been well studied, there are few cell lines containing an endogenous mutation in the tetramerization domain of $\mathrm{p} 53$. Here, we report the discovery of a canine mammary gland tumor cell line CTB-m2, which contains the Leu332Gln (L332Q) mutation corresponding to Leu344 in the tetramerization domain of human p53. Although CTB-m2 cells are genetically heterozygous for the Leu332Gln mutation, the mutant mRNA was almost exclusively expressed.
\end{abstract}

Correspondence to: Dr Kazuhiko Ochiai, School of Veterinary Nursing and Technology, Faculty of Veterinary Science, Nippon Veterinary and Life Science University, 1-7-1 Kyonan-Cho, Musashino, Tokyo 180-8602, Japan

E-mail: kochiai@nvlu.ac.jp

Dr Masami Morimatsu, Laboratory of Laboratory Animal Science and Medicine, Department of Disease Control, Graduate School of Veterinary Medicine, Hokkaido University, Kita-18, Nishi-9, Sapporo 060-0818, Japan

E-mail: mmorimat@vetmed.hokudai.ac.jp

*Contributed equally

Key words: canine, mammary gland tumor, mutation, p53, tetramerization
CTB-m2 cells showed enhanced cell proliferation compared to wild-type p53-expressing CTB-m cells of the same lineage. A p53 tetramerization reporter assay showed that the ability of the p53 mutant to form tetramers was significantly lower than that of wild-type p53. An immunoblot analysis of cross-linked p53 oligomerized forms demonstrated that the L332Q mutant lacked the ability to form tetramers but retained the ability to form dimers. These data suggest that the p53 mutant cell line CTB-m 2 could be a useful tool for analyzing the precise tetramerization mechanisms of p53 and verifying the effects of therapeutic agents against tumors expressing p53 mutants that lack the ability to tetramerize.

\section{Introduction}

The tumor suppressor protein p53 plays a central role in the cell cycle and maintenance of genomic integrity (1-3). The p53 gene is frequently mutated in human cancers, and germline mutations are the underlying cause of Li-Fraumeni syndrome $(4,5)$. The human p53 protein is comprised of an $\mathrm{N}$-terminal transactivation domain, a proline-rich region, a structured DNA-binding domain connected to a tetramerization domain (TD) via a flexible linker, and a C-terminal regulatory domain (6). The p53 protein is active as a homotetramer which adopts a dimer of dimer topology (7). The TD in human p53 (amino acid residues 326-356) exhibits a dihedral symmetry of dimers (8). Two monomers interact with the $\beta$-strands (Glu326-Arg333) to form a dimer, and the two dimers then interact as a $\alpha$-helix bundle (Arg335-Gly356) to form the tetramer (9). Nine residues in the TD in human p53 (Phe328, Leu330, Ile332, Arg337, Phe338, Met340, Phe341, Leu344 and Leu348) are critical determinants in stabilizing the p53 tetramer (10). Leu344 mutants lacking the ability to 
dimerize (L344P), or tetramerize (L344R), have been previously reported (11), and notably, germline L344P mutation has been found in a family with Li-Fraumeni syndrome (12). Leu344 is located in the $\alpha$-helix which forms the hydrophobic core of the tetramer interface.

Although, numerous cell lines containing mutated p53 genes have been established from cancer tissues, there are few cell lines which contain a mutated p53 tetramerization domain $(13,14)$. Uyama et al established and characterized four pairs of canine mammary tumor cell lines derived from either primary and metastatic origin (15). One of these cell lines, CTB-m, was passaged 50 times in our laboratory to obtain a line containing a spontaneous L332Q mutation in p53 (corresponding to human L344); we designated this new cell line as CTB-m2. In this study, we assessed the oligomerization abilities of this canine p53 L332Q mutant and performed a tetramerization reporter assay.

\section{Materials and methods}

Cell culture and transfection. The CIP-p, CIP-m, and CTB-m cell lines were kindly provided by Dr N. Sasaki of the University of Tokyo. CIP-p, CIP-m, CTB-m and CTB-m 2 cells were cultured in RPMI-1640 medium (Wako, Osaka, Japan) and HeLa cells (ATCC, Rockville, MD, USA) were cultured in DMEM (Wako). All media were supplemented with $10 \%$ fetal bovine serum (FBS) and penicillin-streptomycin (Wako) and cells were cultured under a humidified atmosphere of $5 \% \mathrm{CO}_{2}$ at $37^{\circ} \mathrm{C}$. Cell transfection was performed using FuGENE HD (Promega, Madison, WI, USA), as previously reported (16).

Establishment of the CTB-m2 cell line. CTB-m2 cells were spontaneously established from the CTB-m line after 50 passages in our laboratory.

Sequencing analysis and cloning of the canine p53 gene. Total RNA from CTB-m and CTB-m2 cells was obtained using a High Pure RNA Tissue Kit (Roche Diagnostics, Mannheim, Germany), and reverse transcription conducted using a Reverse Transcription System (Promega) according to the manufacturer's protocol. Full length canine p53 cDNA was amplified by PCR using the following primers: 5'-CTCGAGGACCAC CATGCAAGAGCCACAGTCAGAGC-3' and 5'-GAATTCCC GTCTGAGTCGGGCCCTTCTCTC-3'. Following digestion with $X h o I$ and $E c o$ RI, the PCR product was cloned into the XhoI and EcoRI sites of the plasmid pMACS KK HA(C) (Miltenyi Biotec, Bergisch Gladbach, Germany). Samples containing genomic DNA from cells were prepared using the ZR Genome DNATM Tissue Miniprep (Zymo Research, Irvine, CA, USA). Exon 10-11 of p53 containing the sequence encoding L332 was amplified by PCR using the following primers; 5'-GCACTTACACCTTAGTCTGAG-3', and 5'-CGG AATAGGTGTGCTCAAGC-3'. The amplified canine p53 cDNA and exon 10-11 were directly sequenced using an ABI 3730 system (Applied Biosystems, Waltham, MA, USA).

Cell proliferation analysis. A total of 20,000 cells were plated into each well of a 24 -well plate, and counted every $24 \mathrm{~h}$ up until 72 h using a TC20 ${ }^{\mathrm{TM}}$ Automated Cell Counter (Bio-Rad, Hercules, CA, USA). p53 tetramerization reporter assay. The p53 response element in p21WAF (GenBank accession no. U24170, nucleotides 2303-2321) and human cytomegalovirus in the pTet-splice vector (nucleotides 318-446; Clontech Laboratories Inc., Palo Alto, CA, USA) (17) was inserted between the XhoI and HindIII sites in pNL1.1[Nluc] (p53RE-pNL1.1) (Promega). To measure the endogenous p53 tetramerization activities, CTB-m and CTB-m2 cells were transfected with the p53RE-pNL1.1 reporter plasmid. The cells were harvested $48 \mathrm{~h}$ after transfection, and luciferase activity was measured using the Nano-Glo Dual-Luciferase Reporter Assay System (Promega). The luciferase activity was normalized to the value of the luc2 activity from co-transfected pGL4.51[1uc2/ $\mathrm{CMV} / \mathrm{Neo}$ (Promega). To measure the exogenous p53 tetramerization activities, HeLa cells were co-transfected with p53RE-pNL1.1 and either WT canine p53 or its L332Q mutant in pMACS KK HA (C).

Sample preparation and cross-linking procedure. HeLa cells in 6-well plates were transfected with either WT or L332Q p53 expression plasmids ( $1 \mu \mathrm{g} /$ well). After $48 \mathrm{~h}$ of transfection, the cells were treated with doxorubicin at a final concentration of $0.5 \mu \mathrm{M}$ for $6 \mathrm{~h}$. Cells were lysed with mammalian lysis buffer (Promega) supplemented with a protease inhibitor cocktail (Promega). After lysis, the samples were centrifuged $\left(15,000 \mathrm{x} \mathrm{g}\right.$ for $15 \mathrm{~min}$ at $\left.4^{\circ} \mathrm{C}\right)$ to obtain the supernatant. Total protein levels were measured using BCA (Nacalai Tesque, Kyoto, Japan). Equal amounts of proteins (100 $\mu \mathrm{g} /$ condition) were incubated with glutaraldehyde at different concentrations $(0,0.02$ or $0.04 \%)$ and incubated on ice for $30 \mathrm{~min}$. To make a working solution of glutaraldehyde, the commercially available $25 \%$ glutaraldehyde solution was diluted in PBS and discarded after use. To quench the reaction, sample buffer was added to obtain the following final concentrations: $250 \mathrm{mM}$ Tris- $\mathrm{HCl}, \mathrm{pH} 8.5 ; 2 \%$ lithium dodecyl sulfate; 100 mM DTT; $0.4 \mathrm{mM}$ EDTA; $10 \%$ glycerol; $0.2 \mathrm{mM}$ bromophenol blue. Samples were then separated by polyacrylamide gel electrophoresis (18).

Electrophoresis and immunoblotting. Samples were resolved on a 5-12.5\% Tris-glycine polyacrylamide gradient gel using a Perfect Cell B from DRC (Tokyo, Japan) $(\Delta \mathrm{V}=150 \mathrm{~V}, 50 \mathrm{~min}$ at $25^{\circ} \mathrm{C}$ ), transferred to a PVDF membrane using a standard semi-dry apparatus from Bio- $\operatorname{Rad}\left(\Delta \mathrm{V}=15 \mathrm{~V}, 1 \mathrm{~h}\right.$ at $\left.25^{\circ} \mathrm{C}\right)$, and probed with an anti-HA specific mouse monoclonal antibody (M180-3, MBL, Aichi, Japan), and then incubated with HRP-conjugated secondary anti-mouse IgG (7076; Cell Signaling Technology, Beverly, MA, USA). Immunoreactive bands were visualized with a gel documentation system (LAS-4000 mini; Fujifilm, Tokyo, Japan).

Crystal structure modeling. We retrieved the crystal structure of the human p53 tetramerization domain from the Research Collaboratory for Structural Bioinformatics Protein Data Bank at http://www.rcsb.org/ (PDB ID: 3FAK) and analyzed it using the University of California, San Francisco (UCSF) Chimera software (http://www.cgl.ucsf.edu/chimera/) (18).

Microsatellite genotyping of canine cell lines by polymerase chain reaction $(P C R)$ and amplified fragment length 
A

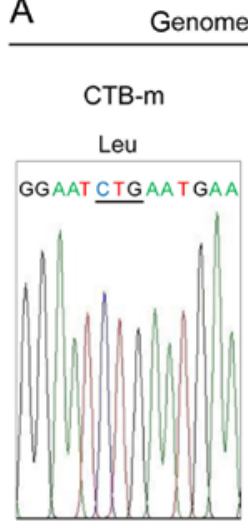

Genome sequence

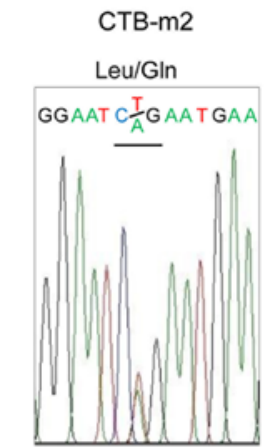

B

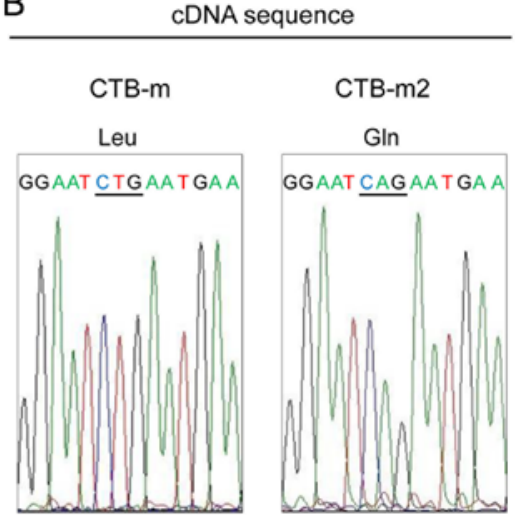

C

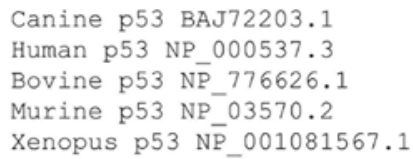

D

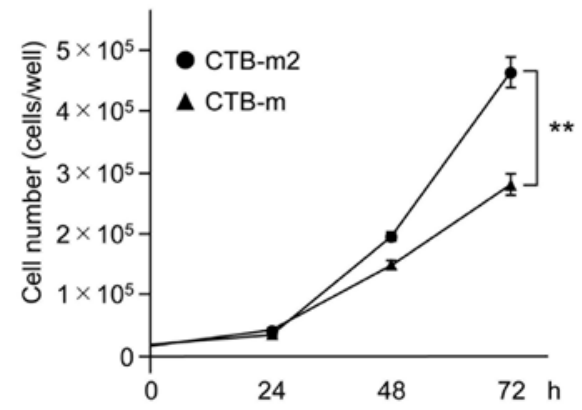

Figure 1. Mutation and cell proliferation analysis of the canine $\mathrm{p} 53 \mathrm{~L} 332 \mathrm{Q}$ mutant cell line CTB-m2. (A) The electropherogram shows the nucleotide substitutions at position c.1151T >A in the canine $p 53$ gene (GenBank accession: AB537893.1) amplified from CTB-m2 genomic DNA (right) and the same position amplified from CTB-m genomic DNA (left). In each case, the nucleotide sequences 1150-1152 encoding Leu332 (CTB-m) or Leu/Gln (CTB-m2) are underlined. (B) Homozygous electropherograms of the $p 53$ gene amplified from either CTB-m or CTBm-2 cDNAs. In each case, the nucleotide sequences 1150-1152 encoding Leu332 (CTB-m) or Gln332 (CTB-m2) are underlined. (C) Amino acid sequence alignments of canine, human bovine, murine and Xenopus p53 proteins (GenBank accession: BAJ72203.1; NP_000537.3; NP_776626.1; NP_03570.2; NP_001081567.1). Canine p53 Leu 332 is homologous to human p53 Leu 344 and other species. The bold font indicates the homology positions. (D) Cell proliferation was assessed in CTB-m or CTB-m 2 cells every $24 \mathrm{~h}$ after passage using a TC20 ${ }^{\mathrm{TM}}$ Automated Cell Counter. Data were obtained from three independent experiments. Values are shown as mean $\pm \mathrm{SD}$. ${ }^{* *} \mathrm{P}<0.01$ (Student's t-test).

polymorphism analysis. Thirteen microsatellite loci were compared between the CTB-m and CTB-m2 or CIP-p and CIP-m cell lines by PCR and amplified fragment length polymorphism analysis in a genetic diagnosis laboratory (http://www.kahotechno.co.jp/clinic/index.html, Kahotechno, Fukuoka, Japan).

Statistical analysis. Data are expressed as mean \pm standard deviation (SD). Differences between two groups were analyzed using the Student's t-test. Analysis of variance (ANOVA) with a Tukey's post hoc test was used when multiple comparisons were required. Significance was assessed at the 0.05 (or lower) level for all tests.

\section{Results}

p53 in CTB-m2 cells contains a heterozygous mutation at $L 332 Q$. Sequencing analysis of genomic $p 53$ from the canine mammary gland tumor cell line CTB-m2 showed the presence of a heterozygous missense mutation $\mathrm{L} 332 \mathrm{Q}$, compared to
CTB-m cells, which expressed homozygous L332 (Fig. 1A). However, the sequence of $p 53$ mRNA derived from CTB-m2 cells showed almost exclusively the mutated allele (L332Q) (Fig. 1B). A comparison of a part of the tetramerization domain of the canine 553 protein (GenBank accession: BAJ72203.1) with human, bovine, murine and Xenopus p53 protein (NP_000537.3; NP_776626.1;NP_03570.2; NP_001081567.1) showed that canine Leu332 corresponds to human Leu344, and that this sequence is completely conserved in other species (Fig. 1C).

Cell viability of CTB-m2 cells is higher than CTB- $m$. A cell proliferation analysis was performed using an automatic cell counter every $24 \mathrm{~h}$ after cultivation. Seventy-two hours after cultivation, CTB-m2 cell proliferation was found to be significantly higher than CTB-m cell proliferation (Fig. 1D).

L332Q mutant in p53 reduces the tetramerization ability compared with WT $p 53$. The p53 tetramer response element, along with the human cytomegalovirus minimal promoter sequence, were cloned upstream of the sequence encoding 

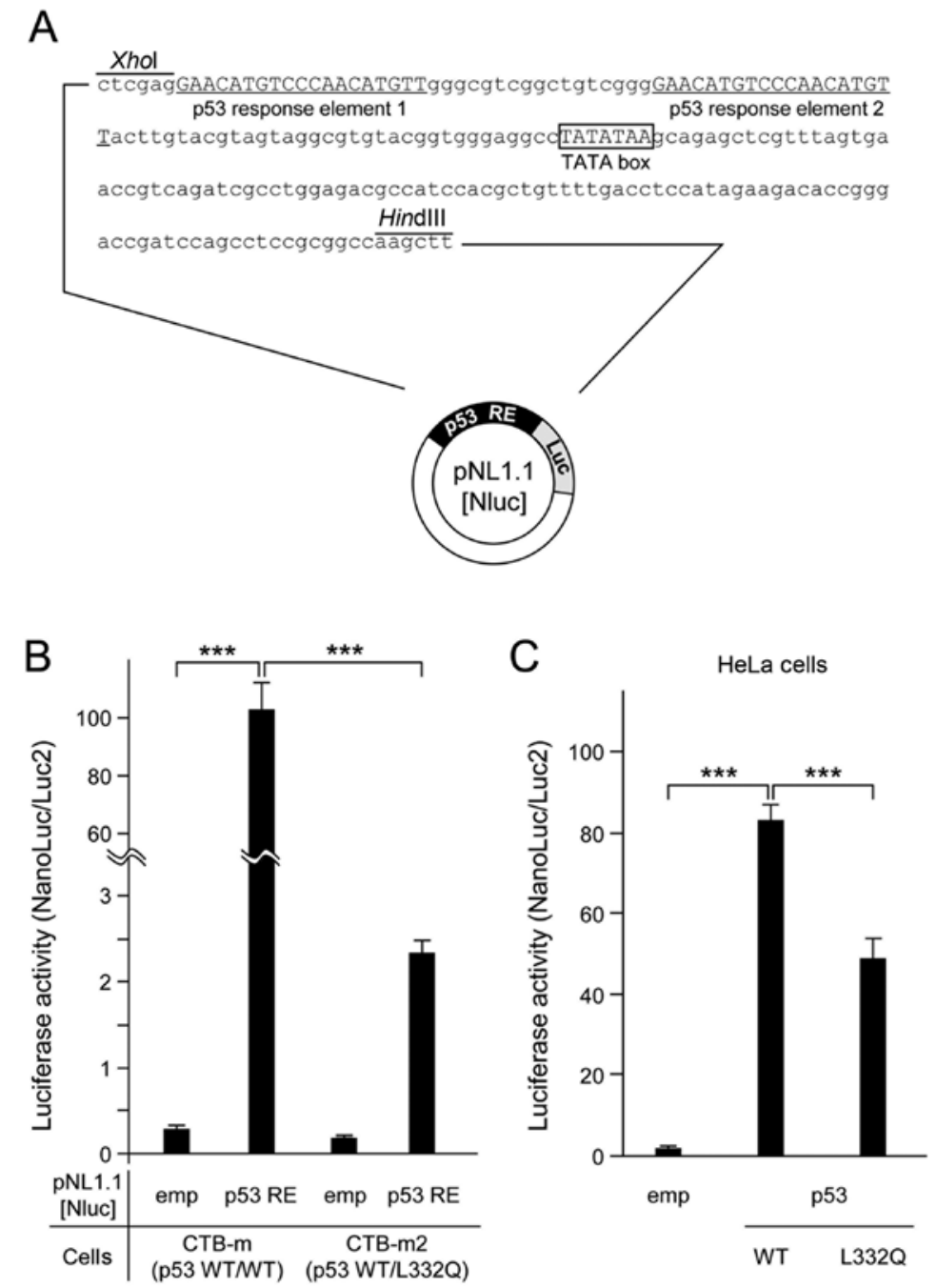

Figure 2. Tetrameric p53-dependent transcriptional activity assessed using a luciferase reporter system. (A) The reporter vector, p53RE-hCMV1-Nluc, was constructed by inserting the p53-responsive elements from $p 21^{\text {WAFI }}$ (shown in the underlined uppercase letters) and the hCMV minimal promoter region, between $X h o$ I and HindIII sites in pNL1.1[Nluc]. The TATA box is shown by boxed lines. The luciferase activities which reflect the p53 tetramerization ability of endogenous p53 in CTB-m or CTB-m2 cells (B), or the tetramerization ability of canine p53 WT, or its L332Q mutant, ectopically expressed in HeLa cells $(C)$ were assessed by the luciferase reporter assay. Data represent the mean of three independent experiments. Values are shown as mean \pm SD. Asterisks atop the brackets indicate significant differences calculated by ANOVA with Tukey's multiple-comparison test $\left({ }^{* * *} \mathrm{P}<0.001\right)$. WT, wild-type.

Nano Luc (Fig. 2A), and the resulting plasmid was transfected into CTB-m or CTB-m 2 cells, or into HeLa cells also expressing exogeneous p53 WT (wild-type) or its L332Q mutant, in order to assess endogenous or exogenous $\mathrm{p} 53$ tetramerization. The L332Q heterozygous mutant CTB-m2 had an approximately 50-fold reduction in luciferase activity compared to WT CTB-m cells (Fig. 2B). Forced expression of the canine p53 L332Q mutant in HeLa cells also showed significantly lower luciferase activity compared to forced expression of WT p53 (Fig. 2C).

The p53 L332Q mutant lacks the ability to tetramerize but not the ability to dimerize. The oligomerization ability of p53 transfected into HeLa cells was assessed using a glutaraldehyde cross-linking assays. Cell lysates from HeLa cells expressing HA-tagged WT p53 or HA-tagged p53 L332Q were treated with 0.02 and $0.04 \%$ glutaraldehyde crosslinker and then analyzed by western blotting. The HA-tagged WT p53 formed both dimers and tetramers following glutaraldehyde treatment. On the other hand, the L332Q mutant formed only a dimer (Fig. 3).
The L332Q mutation results in a conformational change similar to that observed for the L344R mutation in human $p 53$. To verify the results of the functional assay, the protein structure editing tool in the UCSF Chimera software package was used to analyze the possible structural outcomes of L332Q substitutions and L344P(R) mutations based on previous reports (11). The L344 of chain a showed hydrogen bonds with amino acids belonging to the intra-strand and L344/L348 in chain $\mathrm{b}$ (Fig. 4A). The Rotamers tool allows amino acid side chain rotamers to be viewed and evaluated (19). The best rotamers for Arg, Pro, or Gln were selected based on their side-chain torsion, as well as probability values in the rotamer library and in the context of the structural environment. These calculations revealed that the substitution L344P disrupted the inter-strand hydrogen bond required for tetramerization (Fig. 4B). The L344R and L344Q substitutions resulted in a disruption of the hydrogen bonds to chain $b$, and with appearance of hydrogen bonds to the substituted R344 or Q344 and M340 in chain c. The L344R mutant also showed contact with R344 in chain d (Fig. 4C and D). 


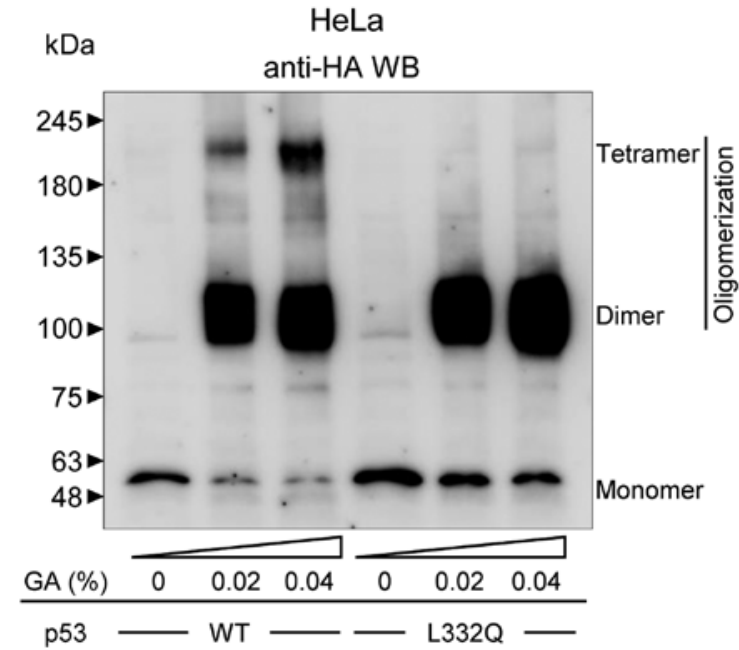

Figure 3. Glutaraldehyde cross-linking analysis of WT and the p53 L332Q mutant. HeLa cells were transfected with plasmids encoding either HA-tagged canine WT or L332Q p53. Cell lysates from the transfected cells were incubated on ice in the presence of the indicated glutaraldehyde (GA) concentrations for $30 \mathrm{~min}$, following which the lysates were separated by polyacrylamide gel electrophoresis and immunoblotted with an anti-HA antibody. Both p53 (WT and L332Q) showed dimer formation after being treated with GA, but tetramerization was not observed for the p53 L332Q mutant. WT, wild-type.
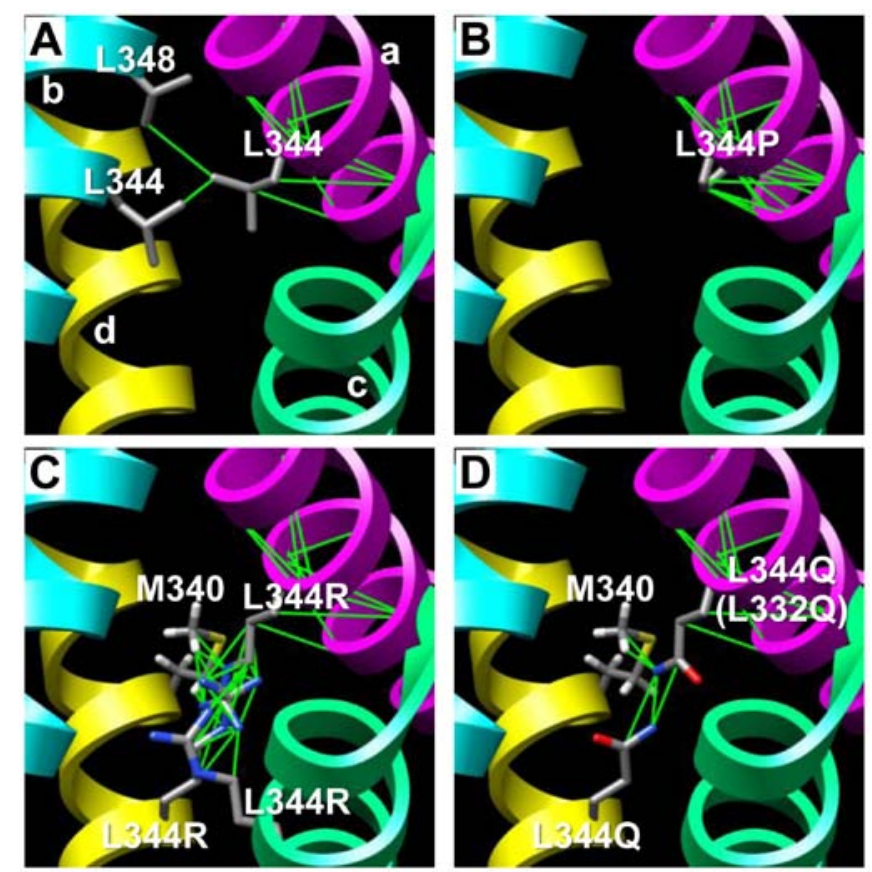

Figure 4. Effect of the L332Q mutation of canine $\mathrm{p} 53$ on its oligomerization. The crystal structure of the human 553 tetramerization domain (PDB ID: 3FAK) was visualized using the Chimera software from UCSF. L344 (corresponding to L332 in canine p53) was mutated to Pro (P), Arg (R), or Gln (Q) residues using the Dunbrack backbone-dependent rotamer library algorithm in the Chimera software from UCSF. Contacts between the residue at position 344 in chain a were calculated and are depicted for (A) L344, (B) L344P, (C) L344R, and (D) L344Q. Solid green lines signify stable contacts, as determined by the Chimera program.

Microsatellite analysis of cell lines established from the same origins. Thirteen microsatellite loci were compared among the CTB or CIP canine mammary gland tumor cell lines.
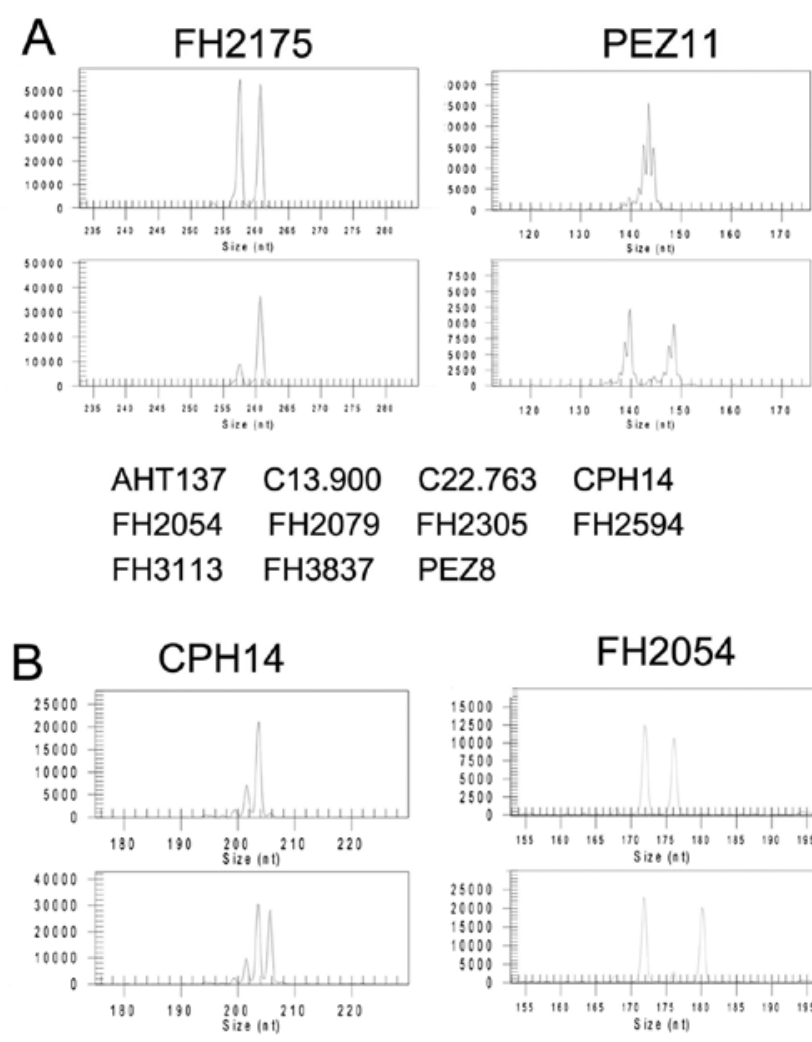

$\mathrm{FH} 2054$
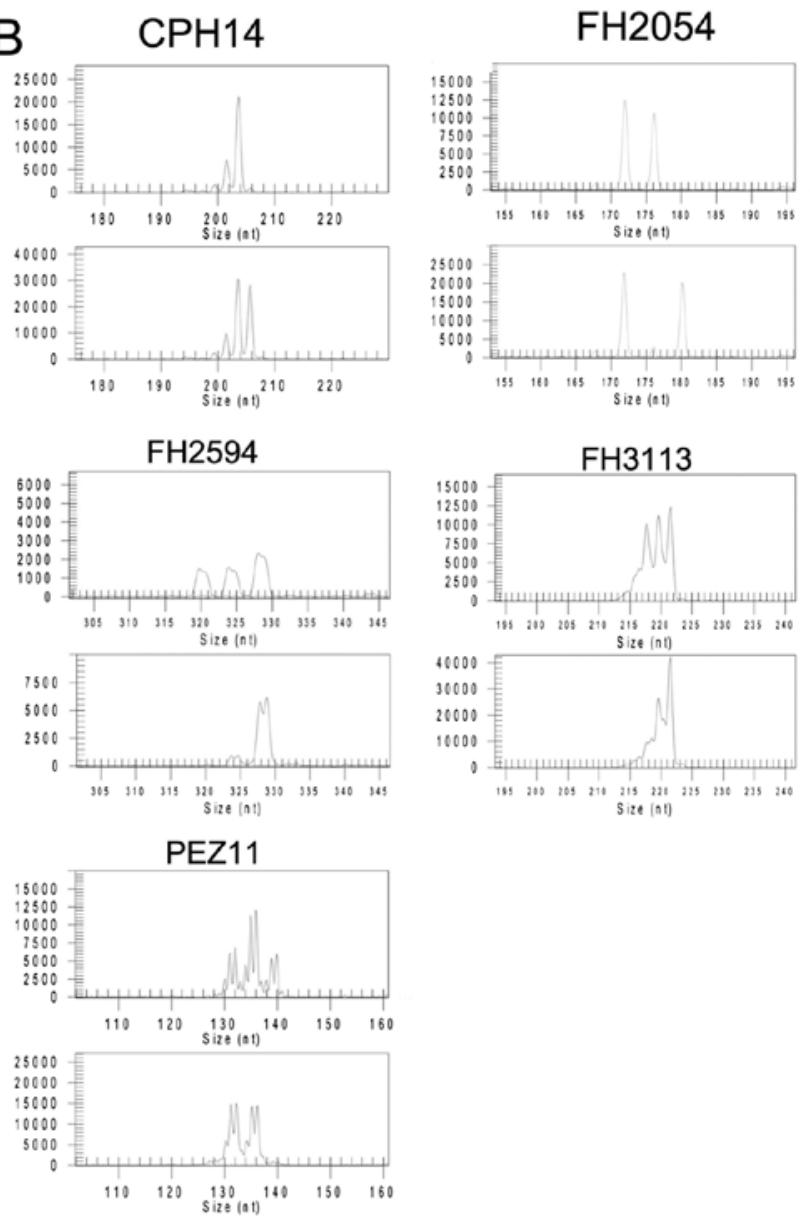
AHT137
C13.900
$\mathrm{C} 22.763 \quad \mathrm{FH} 2079$
$\mathrm{FH} 2175$
$\mathrm{FH} 2305$
$\mathrm{FH} 3837$
PEZ8

Figure 5. Microsatellite analysis of cell lines established from the same origins. (A) Thirteen microsatellite loci were compared between CTB-m (upper panel) and CTB-m2 (lower panel) cells. Two loci (FH2175 and PEZ11) were different, but the other 11 loci shown under the panels were identical between these two cell lines. (B) Comparison between CIP-p (upper panel) and CIP-m (lower panel). Five (CPH14, FH2054, FH2594, FH3113, and PEZ11) of the 13 loci were found to be different between these two cell lines. The other 8 loci shown under the panels were identical between these two cell lines.

Two microsatellite loci between the CTB-m and CTB-m2 cell lines were different, while the other 11 were identical. Five microsatellite loci between the CIP-p and CIP-m cell lines were different, while the other 8 were identical (Fig. 5A and B). 


\section{Discussion}

The functions of the tetramerization domain in the p53 protein and its inhibitory and stabilizing ligands have been well investigated using biochemical, cell biological and in silico analysis (20-22). However, there were no suitable models expressing a mutant of p53 containing a dysfunction in the tetramerization domain. The NCI-H1299 cell line, which does not express the p53 protein, has typically been used to investigate the effect of exogenous p53 mutant expression $(23,24)$. Here, we established the cell line CTB-m2 which expresses the p53 L332Q (which corresponds to L344 in human p53) mutant. Microsatellite analysis using 11 markers showed that in CTB-m cells, which are the parental cells for CTB-m2, are nearly identical compared with another pair of canine mammary gland tumor cell lines, namely CIP-p and CIP-m (Fig. 5A and B) (15). These data suggest that the L332Q mutation in CTB-m2 is a post-oncogenic transformation. Despite being genetically heterozygous at the nucleotides encoding L332, CTB-m2 cells expressed almost only the mutated L332Q p53 mRNA suggesting that in these cells the wild-type p53 allele is inactive. The expression of p53 mutants has been shown to be heterogenous and gene methylation has also been observed in human cancers $(25,26)$. CTB-m 2 cells may therefore have a unique expression status among p53 mutant alleles. Leu332 in canine p53 corresponds to Leu344 in human p53, which is widely recognized as being an essential residue for tetramerization (10). A cell proliferation analysis of the mutated p53 showed enhanced viability of CTB-m2 cells compared with CTB-m cells. The p53 R280T mutant, which is located in the DNA binding domain also promoted cell proliferation in a human glioma cell line (27). Ablation of p53 tetramerization as a result of the L332Q mutation would be expected to change the conformation of the DNA binding domains, which may then result in enhancement of cell proliferation (28).

We examined the tetramerization ability of the p53 L332Q mutant using a functional reporter assay. Two p53 response elements and the minimal human cytomegalovirus promoter sequence, cited by a previous study (17), were modified and cloned into a vector containing a luciferase reporter. The large difference in luciferase activities observed between CTB-m and CTB-m 2 cells, which reflects the endogenous tetramerization abilities of p53 in these two cells, suggest that the L332Q mutation is critical for tetramerization, and also confirms that almost only the mutated allele is expressed in CTB-m 2 cells. Although there was a significant attenuation in tetramerization for the p53 L332Q mutant, the dimer forming ability of this mutant was retained, as shown by electrophoresis and immunoblotting of p53 cross-linked with glutaraldehyde. The in silico simulation of amino acid substitution supports this retention of dimerization ability, and L332Q (L344Q) mutant showed a greater degree of similarity to L344R, which dimerizes, than to L344P, which can only form monomers. The mutated residues in the L332Q (L344Q), L344P, and L344R mutants could not form bonds with Leu344 and Leu348 of the neighboring chain in the tetramerization domain. However, both L332Q (L344Q) and L344R mutants showed contacts with Met340 and L344Q (L344R) of the oblique neighboring chain.
In summary, we established and characterized a new cell line, CTB-m2, which expresses p53 L332Q, a mutant in the p53 tetramerization domain. As a result of this mutation, p53 L332Q lacked tetramerization but not dimerization ability. These CTB-m 2 cells can be used to investigate p53 pathogenesis and to evaluate new strategies to restore p53 function (29).

\section{Acknowledgements}

Not applicable.

\section{Funding}

The present study was supported by the KAKENHI Scientific Research grants from the Ministry of Education, Culture, Sports, Science and Technology of Japan (nos. 15K07754 and 26450400).

\section{Availability of data and materials}

The analyzed data sets generated during the study are available from the corresponding author upon reasonable request.

\section{Authors' contributions}

$\mathrm{KO}, \mathrm{MM} 1$ and TO designed the research. KO and DA mainly did the research. HH, SK, MM2, AE and TS also performed some experiments. TN and MW analyzed the data. All authors read and approved the manuscript and agree to be accountable for all aspects of the research in ensuring that the accuracy or integrity of any part of the study are appropriately investigated and resolved.

\section{Ethics approval and consent to participate}

Not applicable.

\section{Consent for publication}

Not applicable.

\section{Competing interests}

The authors declare that they have no competing interests.

\section{References}

1. Meek DW: Tumour suppression by p53: A role for the DNA damage response? Nat Rev Cancer 9: 714-723, 2009.

2. Lane D and Levine A: p53 Research: The past thirty years and the next thirty years. Cold Spring Harb Perspect Biol 2: a000893, 2010.

3. Bieging KT, Mello SS and Attardi LD: Unravelling mechanisms of p53-mediated tumour suppression. Nat Rev Cancer 14: 359-370, 2014.

4. Petitjean A, Mathe E, Kato S, Ishioka C, Tavtigian SV, Hainaut P and Olivier M: Impact of mutant p53 functional properties on TP53 mutation patterns and tumor phenotype: Lessons from recent developments in the IARC TP53 database. Hum Mutat 28: 622-629, 2007.

5. Olivier M, Hollstein M and Hainaut P: TP53 mutations in human cancers: Origins, consequences, and clinical use. Cold Spring Harb Perspect Biol 2: a001008, 2010.

6. Joerger AC and Fersht AR: Structural biology of the tumor suppressor p53. Annu Rev Biochem 77: 557-582, 2008. 
7. Rajagopalan S, Huang F and Fersht AR: Single-molecule characterization of oligomerization kinetics and equilibria of the tumor suppressor p53. Nucleic Acids Res 39: 2294-2303, 2011.

8. Jeffrey PD, Gorina S and Pavletich NP: Crystal structure of the tetramerization domain of the p53 tumor suppressor at 1.7 angstroms. Science 267: 1498-1502, 1995.

9. Kamada R, Toguchi Y, Nomura T, Imagawa T and Sakaguchi K Tetramer formation of tumor suppressor protein p53: Structure, function, and applications. Biopolymers 106: 598-612, 2016.

10. Mateu MG and Fersht AR: Nine hydrophobic side chains are key determinants of the thermodynamic stability and oligomerization status of tumour suppressor p53 tetramerization domain. EMBO J 17: 2748-2758, 1998.

11. Kamada R, Nomura T, Anderson CW and Sakaguchi K: Cancerassociated p53 tetramerization domain mutants: Quantitative analysis reveals a low threshold for tumor suppressor inactivation. J Biol Chem 286: 252-258, 2011.

12. Varley JM, Thorncroft M, McGown G, Tricker K, Birch JM and Evans DG: A novel deletion within exon 6 of TP53 in a family with Li-Fraumeni-like syndrome, and $\mathrm{LOH}$ in a benign lesion from a mutation carrier. Cancer Genet Cytogenet 90: 14-16, 1996.

13. Atema $A$ and Chène $P$ : The gain of function of the $p 53$ mutant Asp281Gly is dependent on its ability to form tetramers. Cancer Lett 185: 103-109, 2002.

14. Muller PA and Vousden KH: Mutant $\mathrm{p} 53$ in cancer: New functions and therapeutic opportunities. Cancer Cell 25: 304-317, 2014

15. Uyama R, Nakagawa T, Hong SH, Mochizuki M, Nishimura R and Sasaki N: Establishment of four pairs of canine mammary tumour cell lines derived from primary and metastatic origin and their E-cadherin expression. Vet Comp Oncol 4: 104-113, 2006.

16. Kato Y, Ochiai K, Kawakami S, Nakao N, Azakami D, Bonkobara M, Michishita M, Morimatsu M, Watanabe M and Omi T: Canine REIC/Dkk-3 interacts with SGTA and restores androgen receptor signalling in androgen-independent prostate cancer cell lines. BMC Vet Res 13: 170, 2017

17. Imagawa T, Terai T, Yamada Y, Kamada R and Sakaguchi K Evaluation of transcriptional activity of p53 in individual living mammalian cells. Anal Biochem 387: 249-256, 2009.

18. Cubillos-Rojas M, Schneider T, Sánchez-Tena S, Bartrons R, Ventura F and Rosa JL: Tris-acetate polyacrylamide gradient ge electrophoresis for the analysis of protein oligomerization. Anal Bioanal Chem 408: 1715-1719, 2016.
19. Pettersen EF, Goddard TD, Huang CC, Couch GS, Greenblatt DM, Meng EC and Ferrin TE: UCSF Chimera - a visualization system for exploratory research and analysis. J Comput Chem 25: $1605-1612,2004$

20. Salvatella X, Martinell M, Gairí M, Mateu MG, Feliz M, Hamilton AD, De Mendoza J and Giralt E: A tetraguanidinium ligand binds to the surface of the tetramerization domain of protein P53. Angew Chem Int Ed Engl 43: 196-198, 2004.

21. Martinell M, Salvatella X, Fernández-Carneado J, Gordo S, Feliz M, Menéndez M and Giralt E: Synthetic ligands able to interact with the 553 tetramerization domain. Towards understanding a protein surface recognition event. ChemBioChem 7: 1105-1113, 2006

22. Gordo S, Martos V, Santos E, Menéndez M, Bo C, Giralt E and de Mendoza J: Stability and structural recovery of the tetramerization domain of p53-R337H mutant induced by a designed templating ligand. Proc Natl Acad Sci USA 105: 16426-16431, 2008.

23. Osaki S, Nakanishi Y, Takayama K, Pei XH, Ueno H and Hara N: Alteration of drug chemosensitivity caused by the adenovirus-mediated transfer of the wild-type p53 gene in human lung cancer cells. Cancer Gene Ther 7: 300-307, 2000.

24. Yoshikawa K, Hamada J, Tada M, Kameyama T, Nakagawa K, Suzuki Y, Ikawa M, Hassan NM, Kitagawa Y and Moriuchi T: Mutant p53 R248Q but not R248W enhances in vitro invasiveness of human lung cancer NCI-H1299 cells. Biomed Res 31: 401-411, 2010.

25. Tornaletti S and Pfeifer GP: Complete and tissue-independent methylation of $\mathrm{CpG}$ sites in the p53 gene: Implications for mutations in human cancers. Oncogene 10: 1493-1499, 1995.

26. Soussi T and Lozano G: p53 mutation heterogeneity in cancer. Biochem Biophys Res Commun 331: 834-842, 2005.

27. Lin C, Liang Y, Zhu H, Zhang J and Zhong X: R280T mutation of p53 gene promotes proliferation of human glioma cells through GSK-3ß/PTEN pathway. Neurosci Lett 529: 60-65, 2012.

28. Retzlaff M, Rohrberg J, Küpper NJ, Lagleder S, Bepperling A, Manzenrieder F, Peschek J, Kessler H and Buchner J: The regulatory domain stabilizes the p53 tetramer by intersubunit contacts with the DNA binding domain. J Mol Biol 425: 144-155, 2013.

29. Joerger AC and Fersht AR: The p53 Pathway: Origins, inactivation in cancer, and emerging therapeutic approaches. Annu Rev Biochem 85: 375-404, 2016. 